\title{
Resistência de clones de batata a Bemisia tabaci biótipo B
}

\author{
Artur BO Rocha ${ }^{1}$; André L Lourenção ${ }^{1}$; Hilário S Miranda Filho ${ }^{1}$; Pedro CR Hayashi ${ }^{2}$ Valdir J Ramos ${ }^{3}$

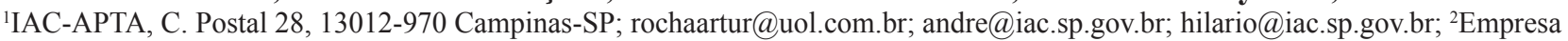 \\ Agropommet, Rod. SP 215 km 34, 13880-000 Vargem Grande do Sul-SP; jarril@uol.com.br; ${ }^{3}$ APTA Regional Sudoeste Paulista, C. \\ Postal 62, 18300-970 Capão Bonito-SP; vramos@apta.sp.gov.br
}

\section{RESUMO}

Avaliou-se a resistência de 30 genótipos de batata a $B$. tabaci (Genn.) biótipo B (Hemiptera: Aleyrodidae) em casa de vegetação, por meio de três experimentos. No primeiro com chance de escolha, foi em blocos ao acaso, 30 tratamentos e três repetições. Avaliou-se a atratividade para adultos, a preferência para oviposição e a densidade de tricomas simples e glandulares. Os sete genótipos com menor oviposição, juntamente com o padrão de resistência 'Achat', e o mais ovipositado foram selecionados para os experimentos seguintes, nos quais se avaliou a preferência para oviposição sem chance de escolha e para o acompanhamento do período de desenvolvimento ovo-adulto, ambos em blocos ao acaso, nove tratamentos e cinco repetições. $\mathrm{Na}$ avaliação de atratividade, após 72 horas, HPC 5B e BAP 82 tiveram o menor número de adultos, enquanto que NYL 235-4 e MAC 2 foram os mais atrativos. Neste mesmo experimento, os genótipos HPC 6R, BACH 4, Clone APTA 2135, HPC 9B, BAP 82, 'Baraka' e HPC 5B foram os menos ovipositados. O genótipo NYL 235-4 mostrou alta densidade de tricomas simples (TS) e glandulares (TG), enquanto os genótipos CH 2, 'Santé' e 'Caesar' tiveram alta densidade de TS, e HPC 9B, 'Radosa', 'Cupido', 'Caesar', 'Saginaw Gold', BACH 3 e HPC 1B para TG revelaram menor pilosidade. Houve correlação significativa entre atratividade para adultos e preferência para oviposição. No experimento sem chance de escolha, os genótipos BACH 4, HPC 9B, 'Baraka' e 'Achat' foram menos ovipositados, caracterizando-se como portadores de resistência do tipo não-preferência para oviposição. Quanto ao período de desenvolvimento ovo-adulto, as médias não diferiram entre os genótipos, variando de 34,4 a 36,6 dias. Observou-se para os genótipos BAP 82, 'Baraka' e $\mathrm{BACH} 4$ o menor número de adultos emergidos, sugerindo a presença de antibiose. Com base nos três experimentos, os clones 'Baraka' e BACH 4 destacaram-se como os mais resistentes a $B$. tabaci biótipo B.

Palavras-chave: Solanum tuberosum, Aleyrodidae, mosca-branca, resistência de plantas a insetos.

\section{ABSTRACT}

\section{Resistance of potato clones to Bemisia tabaci biotype B}

The resistance of 30 potato genotypes to $B$. tabaci (Genn.) biotype B (Hemiptera: Aleyrodidae) was evaluated in three greenhouse experiments. In the first experiment we evaluated the attractiveness, the preference for oviposition and the trichome density in a freechoice test in randomized blocks design with 30 treatments and three replications. In other experiment, no-choice preference for oviposition was evaluated (randomized blocks, nine treatments and five replications). The whitefly egg-adult cycle was monitored using a statistical design in randomized blocks with five replications. In the free-choice test, the genotypes NYL 235-4 and MAC 2 were the most attractive to adults, whereas HPC $5 \mathrm{~B}$ and BAP 82 presented the lowest number of adults. The genotypes HPC 6R, BACH 4, clone APTA 2135, HPC 9B, BAP 82, 'Baraka' and HPC 5B presented the lowest number of eggs. Clone NYL 235-4 had the greatest number of simple (ST) and glandular (GT) trichomes, whereas clone $\mathrm{CH} 2$ had the lowest number of ST, and HPC 5B, 'Radosa', 'Cupido', 'Caesar', 'Saginaw Gold', BACH 3 and HPC 1B of GT. There was significant correlation between adult attractiveness and oviposition preference. In the no-choice test, only the genotypes BACH 4, HPC 9B, 'Baraka and 'Achat' remained resistant. Consequently, for these four genotypes non-preference is the oviposition resistance mechanism. The egg adult cycle varied from 34.4 to 36.6 days. For the adult emergence, genotypes BAP 82, 'Baraka' and BACH 4 showed the lowest number of adults emerged, suggesting the presence of antibiosis. Genotypes BAP82, 'Baraka' and BACH 4 were resistant to B. tabaci biotype B.

Keywords: Solanum tuberosum, Aleyrodidae, silverleaf whitefly, host plant resistance.

\section{(Recebido para publicação em 17 de dezembro de 2010; aceito em 24 de janeiro de 2012) (Received on December 17, 2010; accepted on January 24, 2012)}

$\mathrm{N}$ as últimas décadas, vários cultivos agrícolas de regiões tropicais e subtropicais têm sido severamente atacados pela mosca-branca (Bemisia tabaci), que deixou o status de praga secundária do passado e tornou-se uma das pragas mais importantes da agricultura mundial (Brown, 1994). O biótipo B de B. tabaci foi introduzido no Brasil provavelmente através de plantas ornamentais, sendo registrado pela primeira vez em São Paulo no início da década de 90 (Lourenção \& Nagai, 1994) e posteriormente no Distrito Federal em 1993 (França et al., 1996).

De acordo com Lara et al. (2004a), o cultivo da batata nos trópicos é um dos que mais sofre com problemas fitossanitários decorrentes de ataques de patógenos e insetos, com destaque para a mosca-branca B. tabaci biótipo B. Altas infestações desse inseto em lavouras de batata são relativamente recentes, tendo sido constatados os primeiros surtos populacionais apenas a partir de 2001 (Lourenção et al., 2003). Os danos de B. tabaci biótipo $\mathrm{B}$ à cultura da batata compreendem sucção de seiva, desenvolvimento de fumagina e transmissão de vírus [Tomato yellow vein streak virus (ToYVSV) e Potato leafroll virus (PLRV)] (Lima \& Lara, 2001; SouzaDias et al., 2005). 
O uso de plantas resistentes ao ataque de insetos é uma importante estratégia de manejo, configurando-se como uma solução prática, de baixo custo e longa duração na manutenção de baixas populações de B. tabaci biótipo B, reduzindo assim as perdas na produção (Bellotti \& Arias, 2001).

De acordo com Painter (1968), existem três tipos de resistência a insetos: não-preferência, antibiose e tolerância. A não-preferência usualmente é determinada permitindo-se que a praga escolha entre diferentes genótipos aquele menos favorável para seu abrigo, alimentação e/ou oviposição. Observando-se como uma planta pode resistir ao ataque de um inseto, percebe-se que, na maioria das vezes, isto implica em alterações no comportamento ou na biologia do inseto, ou ainda na reação da própria planta que em nada afeta o inseto que nela se alimenta. $\mathrm{O}$ efeito adverso que uma planta exerce sobre a biologia do inseto pode ser devido à presença de substâncias tóxicas e/ou inibidoras de desenvolvimento (denominadas de antibióticas). Pode, ainda, ser devido a um desequilíbrio nutricional e/ou ausência de nutrientes essenciais ou não, sendo este tipo de resistência denominado antibiose. Este é o tipo de resistência mais desejável, porque geralmente reduz o número de indivíduos da geração seguinte, mantendo a praga em níveis populacionais abaixo do nível crítico. A tolerância é o mecanismo mais difícil de ser quantificado, porque, basicamente envolve comparação de certo número de insetos e o dano subseqüente na planta.

No caso específico da batata, Silva et al. (2008) avaliaram a resistência de 24 clones de ampla variabilidade genética a essa mosca-branca e detectaram apenas um genótipo (Achat) com resistência moderada. Como esse clone é totalmente estéril, não pode ser usado em hibridações em programas de melhoramento que visem à obtenção de cultivares resistentes à mosca-branca. Assim, diante da necessidade de novas avaliações de acessos de batata de genealogias diferentes quanto ao comportamento em relação a $B$. tabaci biótipo $\mathrm{B}$, realizou-se o presente trabalho, que teve como objetivo testar 30 genótipos de batata frente a essa mosca-branca em casa de vegetação, considerando-se a atratividade, a oviposição e a presença de tricomas glandulares e tricomas simples.

\section{MATERIAL E MÉTODOS}

Os experimentos foram conduzidos em casa de vegetação, do IAC em Campinas, durante o período de janeiro a julho de 2010 .

Criação estoque de $B$. tabaci biótipo B - A criação da mosca-branca $B$. tabaci biótipo $\mathrm{B}$ foi conduzida em casa de vegetação $(3 \times 5 \mathrm{~m})$, constituída por base de alvenaria (1 $\mathrm{m}$ de altura), laterais de tela anti-afídeo e teto de vidro, com bancada. Como fontes de alimento foram usadas plantas de soja e couve, trocadas quinzenalmente por outras novas com o propósito de fornecer condições adequadas ao inseto. Periodicamente, plantas de aboboreira (Cucurbita spp.) foram introduzidas na criação para que, com a ocorrência de prateamento das folhas, fosse confirmada a identidade do inseto, uma vez que apenas o biótipo B induz essa desordem fisiológica em aboboreira no Brasil (Brown et al., 1995). Recentemente, os adultos dessa criação foram caracterizados molecularmente, confirmando sua identidade (Fontes et al., 2010).

Seleção dos genótipos de batata - Foram selecionados 30 genótipos de batata (Tabela 1) com genealogia bastante diversa da utilizada por Silva et al. (2008), que estudaram majoritariamente cultivares de Solanum tuberosum ssp tuberosum. Quinze dos genótipos estudados possuem genitores de origem silvestre (Solanum chacoense) ou primitiva (S. phureja e Solanum tuberosum ssp andigena), sendo que na literatura são relatados diversos casos de resistência a insetos para genótipos derivados de S. chacoense (Lara et al., 2004b; Souza et al., 2005; Cooper et al., 2009). Como padrões de resistência e suscetibilidade, foram incluídos os clones 'Achat' e NYL 235-4, respectivamente (Silva et al., 2008).

Teste com chance de escolha Plantas dos 30 genótipos de batata foram mantidas em casa de vegetação, medindo 24 x $8 \mathrm{~m}$, utilizando-se vasos plásticos (3 L), contendo uma planta por vaso. Os vasos foram preenchidos com terra misturada a um composto orgânico à base de casca de amendoim e adubados de acordo com as recomendações para a cultura (Raij et al., 1997). A infestação artificial foi realizada quando as plantas estavam com o primeiro par de folhas completamente desenvolvido, através da introdução de vasos com soja altamente infestada, contendo, em média, 250 adultos de mosca-branca por planta. Os focos de infestação foram colocados de forma equidistante entre os vasos de batata, com um foco para cada quatro vasos.

Após 24, 48 e 72 horas de infestação, sempre no início da manhã, realizou-se a contagem dos adultos presentes na face inferior das duas folhas completamente expandidas de cada planta, por meio de um espelho a fim de não tocar na folha avaliada. Com uma semana de infestação, as duas folhas avaliadas para atratividade foram removidas para a contagem dos ovos presentes na superfície abaxial com auxílio de um microscópio estereoscópico (40x), baseando-se nos procedimentos adotados por Valle \& Lourenção (2002). Após as contagens, determinou-se a área foliar com um medidor de área foliar modelo LI $3100 \mathrm{~A}$ (LI-COR), e posteriormente calculou-se o número de adultos e de ovos por $10 \mathrm{~cm}^{2}$.

O delineamento utilizado foi blocos ao acaso, com 30 tratamentos e três repetições. Cada parcela foi constituída por duas folhas de uma planta. Para análise, as médias de adultos foram transformadas em $(\mathrm{x} / 100)^{1 / 2}$ e de ovos em $\log \mathrm{x}$, sendo comparadas pelo teste de Tukey $(p \leq 0,05)$, utilizando o software estatístico SASM.

Densidade de tricomas glandulares e simples - Para avaliação dos tricomas, utilizaram-se as plantas do experimento com chance de escolha. As folhas foram coletadas quando as plantas tinham o primeiro par de folhas completamente desenvolvido. Foi utilizado microscópio estereoscópico (40x) para as contagens do número de tricomas. Retiraram-se dois folíolos de cada planta, marcando-se na face abaxial de cada folíolo um círculo de $28 \mathrm{~mm}^{2}$ na área inferior e outro na área superior.

Adotou-se o delineamento de blocos ao acaso, com 30 tratamentos e três repe- 
tições. As médias das densidades dos tricomas glandulares foram transformadas em $\log \mathrm{x}$ e as dos tricomas simples não sofreram transformação para análise. As médias foram comparadas pelo teste de Tukey $(p \leq 0,05)$, utilizando o software estatístico SASM.

Teste sem chance de escolha - Para este experimento foram selecionados os sete genótipos com menor oviposição no teste com chance de escolha, juntamente com o controle resistente 'Achat' (Silva et al., 2008), e o mais ovipositado, NYL 235-4. Utilizaram-se vasos (3 L), contendo uma planta cada. Ao redor de cada vaso foi instalada uma armação metálica (35 cm de diâmetro superior x $70 \mathrm{~cm}$ de altura), recoberta por tecido de 'voil'. Os demais procedimentos foram idênticos aos utilizados no ensaio com chance de escolha. Quando as plantas atingiram o primeiro par de folhas completamente desenvolvidas, foi realizada a infestação artificial, introduzindo-se cerca de 200 adultos de $B$. tabaci biótipo B de idade desconhecida em cada vaso. Os adultos foram coletados diretamente das folhas das plantas na criação estoque, por meio de vidros de boca larga, sem sexagem, adotando-se procedimento semelhante ao descrito por Lourenção (1980).

O delineamento experimental foi de blocos ao acaso, com nove tratamentos e cinco repetições. Cada parcela foi constituída de um vaso contendo uma planta. A avaliação foi feita em todas as folhas da planta, uma vez que, conforme demonstrado por Silva et al. (2008), pode haver concentração de ovos nas folhas inferiores. As médias de número de ovos foram transformadas em $\sqrt{\mathrm{x}}$, e submetidas à análise de variância, sendo as médias comparadas pelo teste de Tukey $(\mathrm{p} \leq 0,05)$, utilizando o software estatístico SASM.

Desenvolvimento ovo-adulto - Para a avaliação de biologia, os procedimentos para obtenção das plantas foram os mesmos descritos anteriormente. Quando as plantas atingiram o primeiro par de folhas completamente desenvolvidas, os vasos foram transferidos para o insetário de criação de $B$. tabaci biótipo B por um período de quatro horas para a oviposição do inseto. Após esse período, os adultos foram removidos e as plantas levadas ao laboratório onde, com auxílio de microscópio estereoscópico (40x), realizou-se a demarcação de áreas com 20 ovos cada. A seguir, os vasos foram dispostos em telado com teto plástico e laterais de tela anti-afídeo (4 x 4 m), sobre bancadas de concreto.

Foi feita inspeção diária da área demarcada de cada planta para anotação do número de ovos viáveis, do número de ninfas e do número de pupários vazios (indicativo da emergência de adultos). A partir dessas observações determinou-se o período de desenvolvimento de ovo a adulto e também a porcentagem de emergência. A temperatura média durante o experimento foi determinada com base nos dados da estação meteorológica do CEC.

O delineamento experimental foi de blocos ao acaso, com cinco repetições. Os tratamentos foram os mesmos do experimento de oviposição sem chance de escolha. As médias de ovos e adultos não sofreram transformações e foram comparadas pelo teste de Tukey $(\mathrm{p} \leq 0,05)$, utilizando o software estatístico SASM.

Análises de correlação - A correlação foi determinada através das variáveis estudadas (número de ovos $/ 10 \mathrm{~cm}^{2}$, número de adultos $/ 10 \mathrm{~cm}^{2}$ e densidade de tricomas glandulares e simples), utilizando o software estatístico SASM.

\section{RESULTADOS E DISCUSSÃO}

Teste com chance de escolha - Na avaliação de atratividade após $24 \mathrm{~h}$ (Tabela 1) não houve diferença entre os genótipos. Na avaliação de 48 h, os genótipos com menor número de adultos foram BACH 4 (1,4 adultos/10 $\left.\mathrm{cm}^{2}\right)$ e HPC 5B (2,0 adultos/10 $\left.\mathrm{cm}^{2}\right)$ em contraste com os genótipos MAC 2 e NYL 235-4, que apresentaram a maior média $(13,7)$, ficando os demais em posição intermediária. Após $72 \mathrm{~h}$, os clones HPC $5 \mathrm{~B}\left(1,7\right.$ adultos $\left./ 10 \mathrm{~cm}^{2}\right)$ e BAP $82\left(2,0\right.$ adultos $\left./ 10 \mathrm{~cm}^{2}\right)$ foram os menos atrativos, enquanto que NYL 235-4 (16,7 adultos/10 $\left.\mathrm{cm}^{2}\right)$ e MAC 2 $\left(15,9\right.$ adultos $\left./ 10 \mathrm{~cm}^{2}\right)$ continuaram com as maiores médias de adultos.

A alta atratividade verificada em NYL 235-4 nas três avaliações confirma as observações de Silva et al. (2008) para esse clone. As diferenças de atrati- vidade a partir de $48 \mathrm{~h}$ podem ser explicadas por características da planta que afetam o comportamento de $B$. tabaci biótipo B, tais como coloração e, principalmente, disponibilidade de nutrientes ao inseto, conforme discutido por Van Lenteren \& Noldus (1990). Baldin et al. (2009) relatam que a baixa atratividade do genótipo de abobrinha 'Sandy' a $B$. tabaci biótipo B indica a ocorrência de não preferência nessa cultivar e sugerem a existência de fatores repelentes que podem afetar a atratividade e a permanência da mosca-branca sobre suas folhas. Este fato também foi observado em genótipos de soja e feijão (Mansaray et al., 2009) e tomateiro (Jiang et al. 2001).

Quanto à oviposição, os genótipos HPC 6R (3,2 ovos/10 $\left.\mathrm{cm}^{2}\right)$, BACH 4 $\left(3,5\right.$ ovos $\left./ 10 \mathrm{~cm}^{2}\right)$ e clone APTA 2135 $\left(3,5\right.$ ovos $\left./ 10 \mathrm{~cm}^{2}\right)$ revelaram as menores médias de ovos; já REC 4 (28,0 ovos/10 $\mathrm{cm}^{2}$ ), 'Ranger Russet' (28,1 ovos/10 $\left.\mathrm{cm}^{2}\right)$ e NYL 235-4 (48,8 ovos/10 $\left.\mathrm{cm}^{2}\right)$ foram os mais ovipositados (Tabela 2). O genótipo NYL 235-4 obteve a maior média, semelhante ao reportado por Silva et al. (2008), que também verificaram a maior oviposição em teste com chance de escolha para este mesmo genótipo. Chu et al. (2000) descrevem que variações morfológicas das plantas, coloração e idade das folhas, estado nutricional, além de fatores abióticos, como disponibilidade de luz e mudança nas condições ambientais podem influenciar a oviposição de $B$. tabaci biótipo B. No presente trabalho foram observadas diferenças na morfologia dos genótipos de batata, principalmente quanto à espessura das folhas e diâmetro das hastes, o que pode ter influenciado na oviposição de $B$. tabaci biótipo B nos genótipos HPC 6R, .BACH 4 e clone APTA.2135.

Densidade de tricomas glandulares e simples - Quanto aos tricomas glandulares (Tabela 2), os clones 'Achat' (45,0 tricomas/28 $\mathrm{mm}^{2}$ ) e NYL 235-4 $(59,0)$ tiveram as maiores densidades, diferindo de HPC 9B (9,7 tricomas/28 $\left.\mathrm{mm}^{2}\right)$, 'Radosa' $(9,7)$, 'Cupido' $(10,0)$, 'Caesar' (10,3), 'Saginaw Gold' (11,3), BACH 3 (11,3 tricomas/28 $\mathrm{mm}^{2}$ ) e HPC 1B $(13,0)$, que tiveram as menores densidades.

Fernandes et al. (2009) constataram 
Tabela 1. Genótipos de batata e sua genealogia, médias de atratividade em três períodos de observação e oviposição de Bemisia tabaci biótipo B em 30 genótipos de batata em casa de vegetação (potato genotypes and its genealogy, mean attractiveness in three observation and oviposition periods of Bemisia tabaci biotype B on plants of 30 potato genotypes in the greenhouse). Campinas, IAC, 2010.

\begin{tabular}{|c|c|c|c|c|c|}
\hline \multirow{2}{*}{ Genótipo } & \multirow{2}{*}{ Genealogia } & \multicolumn{3}{|c|}{ Atratividade } & \multirow{2}{*}{$\begin{array}{l}\text { Oviposição } \\
\left(\operatorname{ovos} / \mathrm{cm}^{2}\right)^{1,2}\end{array}$} \\
\hline & & $24 h^{1}$ & $48 h^{1}$ & $72 h^{1}$ & \\
\hline HPC 5B & S. phureja $x$ S. chacoense & $2,4 \mathrm{a}$ & $2,0 \mathrm{c}$ & $1,7 \mathrm{c}$ & $7,5 \mathrm{bcd}$ \\
\hline BAP 82 & Híbrido Bannock Russet x Aracy Ruiva & $4,2 \mathrm{a}$ & 3,6 abc & $2,0 \mathrm{bc}$ & $5,7 \mathrm{bcd}$ \\
\hline Cupido & W72-22-446 x Estima & $4,7 \mathrm{a}$ & $3,6 \mathrm{abc}$ & $2,4 \mathrm{abc}$ & 9,2 abcd \\
\hline $\mathrm{BACH} 4$ & Híbrido Bannock Russet x S. chacoense & $1,2 \mathrm{a}$ & $1,4 \mathrm{c}$ & $2,5 \mathrm{abc}$ & $3,5 \mathrm{~cd}$ \\
\hline Pira 25 & Pirassu (S. tuberosum) x Aracy Ruiva & $4,0 \mathrm{a}$ & 3,4 abc & $3,7 \mathrm{abc}$ & $11,3 \mathrm{abcd}$ \\
\hline $\mathrm{HPC} 6 \mathrm{R}$ & S. phureja $x$ S. chacoense & $2,7 \mathrm{a}$ & 3,1 abc & $3,7 \mathrm{abc}$ & $3,2 \mathrm{~d}$ \\
\hline HPC 1B & S. phureja $x$ S. chacoense & $4,5 \mathrm{a}$ & $4,7 \mathrm{abc}$ & $4,1 \mathrm{abc}$ & $13,3 \mathrm{abcd}$ \\
\hline Agria & Quarta x Semlo & $3,7 \mathrm{a}$ & $3,7 \mathrm{abc}$ & $4,3 \mathrm{abc}$ & 20,4 abcd \\
\hline BACH 1 & Híbrido Bannock Russet x S. chacoense & $6,7 \mathrm{a}$ & $6,2 \mathrm{abc}$ & $4,6 \mathrm{abc}$ & $25,8 \mathrm{abc}$ \\
\hline REC 3 & Reich x S. chacoense & $2,2 \mathrm{a}$ & 3,8 abc & $4,6 \mathrm{~b}$ & 8,1 abcd \\
\hline Saginaw Gold & MS 3231-38 x MS 709 & $3,0 \mathrm{a}$ & $2,7 \mathrm{bc}$ & $5,0 \mathrm{~b}$ & $15,2 \mathrm{abcd}$ \\
\hline Daresa & Black 1256 x Maritta & $2,4 \mathrm{a}$ & 3,8 abc & $6,1 \mathrm{~b}$ & $15,0 \mathrm{abcd}$ \\
\hline HPC 9B & S. phureja $x$ S. chacoense & $4,0 \mathrm{a}$ & 5,2 abc & $6,3 \mathrm{~b}$ & 4,7 bcd \\
\hline $\mathrm{CH} 2$ & HPC 7B x S. chacoense & $3,9 \mathrm{a}$ & $4,2 \mathrm{abc}$ & $6,5 \mathrm{~b}$ & $12,4 \mathrm{abcd}$ \\
\hline REC 2 & Reich x S. chacoense & $3,8 \mathrm{a}$ & $3,7 \mathrm{abc}$ & $6,9 \mathrm{~b}$ & 8,1 abcd \\
\hline Radosa & Bintje x MPI 40.1465/6 & $3,5 \mathrm{a}$ & 4,7 abc & $6,9 \mathrm{abc}$ & 20,0 abcd \\
\hline Caesar & Monalisa x Ropta B 1178 & $3,2 \mathrm{a}$ & 6,0 abc & $7,0 \mathrm{abc}$ & 23,9 abcd \\
\hline Achat & Fina $\mathrm{x}$ Rheinhort & $4,5 \mathrm{a}$ & 5,5 abc & $7,1 \mathrm{abc}$ & $15,7 \mathrm{abcd}$ \\
\hline Baraka & SVP 50-358 x Avenir & $4,4 \mathrm{a}$ & $4,2 \mathrm{abc}$ & $7,7 \mathrm{abc}$ & $7,5 \mathrm{bcd}$ \\
\hline Hermes & DDR $5188 \times 163 / 55$ & $5,7 \mathrm{a}$ & 6,3 abc & $8,7 \mathrm{abc}$ & $16,3 \mathrm{abcd}$ \\
\hline HPC 13R & S. phureja $x$ S. chacoense & $5,3 \mathrm{a}$ & 6,9 abc & $9,1 \mathrm{abc}$ & $24,6 \mathrm{abc}$ \\
\hline BACH 3 & Híbrido Bannock Russet x S. chacoense & $3,4 \mathrm{a}$ & $6,4 \mathrm{abc}$ & $10,3 \mathrm{ab}$ & $16,1 \mathrm{abcd}$ \\
\hline Ranger Russet & MS32-38 x Michibonne & $7,2 \mathrm{a}$ & 8,4 abc & $10,7 \mathrm{ab}$ & $28,1 \mathrm{ab}$ \\
\hline Clone APTA 2135 & Ibituaçú x Itararé & $5,6 \mathrm{a}$ & 7,7 abc & $11,0 \mathrm{ab}$ & $3,5 \mathrm{~cd}$ \\
\hline REC 4 & Reich x S. chacoense & $7,9 \mathrm{a}$ & 7,9 abc & $12,2 \mathrm{ab}$ & $28,0 \mathrm{ab}$ \\
\hline Omega & $277 / 58 \times$ Tondra & $2,2 \mathrm{a}$ & $6,9 \mathrm{abc}$ & $12,4 a b$ & 8,5 abcd \\
\hline Santé & Y66-13-636 x SVP AM 66-42 & 4,6 a & 6,9 abc & $12,5 \mathrm{ab}$ & 11,5 abcd \\
\hline BACH 5 & Híbrido Bannock Russet x S. chacoense & $5,3 \mathrm{a}$ & 7,1 abc & $13,2 \mathrm{ab}$ & 18,4 abcd \\
\hline MAC 2 & Maria Bonita x S. chacoense & $7,3 \mathrm{a}$ & $13,7 \mathrm{a}$ & $15,9 \mathrm{a}$ & $20,6 \mathrm{abcd}$ \\
\hline NYL 235-4 & K421-1 x H266-6 & $8,8 \mathrm{a}$ & $13,7 \mathrm{a}$ & $16,7 \mathrm{a}$ & $48,8 \mathrm{a}$ \\
\hline $\mathrm{CV}(\%)$ & & 30,9 & 30,3 & 31,4 & 24,1 \\
\hline
\end{tabular}

*Médias seguidas pela mesma letra na coluna não diferem significativamente entre si pelo teste de Tukey ( $\mathrm{p}<0,05)$; ${ }^{1} \mathrm{Dados}$ originais; Para análise os dados foram transformados em $\operatorname{arcsen}(\mathrm{x} / 100)^{1 / 2} ;{ }^{2}$ Dados originais; Para análise, os dados foram transformadas em log $\mathrm{x}($ original data; means followed by the same letter in the column do not differ significantly from each other by the Tukey test, $\mathrm{p} \leq 0,05 ;{ }^{1}$ Original data. For statistical analysis, data were transformed to $\operatorname{arcsen}(\mathrm{x} / 100)^{1 / 2}$; ${ }^{2}$ Original data. For statistical analysis, data were transformed to log $\left.\mathrm{x}\right)$.

o efeito repelente de tricomas glandulares provenientes do genótipo de tomateiro 1990 (Solanum lycopersicum) a $B$. tabaci biótipo B. Já Know et al. (2006) observaram o efeito destes tricomas em genótipos de batata (Solanum tuberosum) na diminuição da infestação por Spodoptera exigua. De maneira oposta, Medeiros \& Tingey (2006) verificaram que tricomas glandulares de S. berthaultii e dos seus híbridos não afetaram o desenvolvimento e a sobrevivência de Empoasca fabae e Silva et al. (2008) verificaram que tricomas glandulares em genótipos de batata não afetam o desenvolvimento e a sobrevivência de $B$. tabaci biótipo $\mathrm{B}$.

Com relação aos tricomas simples (Tabela 2), os clones 'Ranger Russet' (68), BAP $82(66,3)$, 'Daresa' $(64,7)$ e NYL 235-4 $(64,3)$ tiveram as maiores densidades, enquanto que $\mathrm{CH} 2(13,3)$,
'Santé' (14,7 ), 'Caesar' (18,7), HPC 9B $(23,0)$ e REC $4(26,3)$ revelaram as menores. Silva et al. (2008) também observaram densidade elevada de tricomas simples para o genótipo NYL 235-4.

Preferência para oviposição em teste sem chance de escolha - Os clones menos ovipositados (Tabela 3) foram BACH 4 (2,5 ovos $\left./ \mathrm{cm}^{2}\right)$, HPC 9B $\left(3,0\right.$ ovos $\left./ \mathrm{cm}^{2}\right)$, Baraka $(4,2)$ e Achat $(5,3)$, diferindo de HPC $6 \mathrm{R}(15,5$ ovos/ 
Tabela 2. Médias de densidade de tricomas da superfície abaxial de folíolos de trinta genótipos de batata, em casa de vegetação (trichome density on the abaxial surface of leaflets of 30 potato genotypes in greenhouse). Campinas, IAC, 2010.

\begin{tabular}{|c|c|c|}
\hline Genótipo & Tricomas glandulares $^{1}$ & Tricomas simples $^{1 ; 2}$ \\
\hline HРC 9B & $9,7 \mathrm{c}$ & 23,0 def \\
\hline Radosa & $9,7 \mathrm{c}$ & 42,0 abcdefg \\
\hline Cupido & $10,0 \mathrm{c}$ & 53,0 abcde \\
\hline Caesar & $10,3 \mathrm{c}$ & 18,7 efg \\
\hline Saginaw Gold & $11,3 \mathrm{c}$ & 35,0 abcdefg \\
\hline BACH 3 & $11,3 \mathrm{c}$ & 30,3 bcdefg \\
\hline HPC 1B & $13,0 \mathrm{c}$ & 31,7 abcdefg \\
\hline BAP 82 & $13,3 \mathrm{bc}$ & $66,3 \mathrm{ab}$ \\
\hline Clone APTA 2135 & $14,3 \mathrm{bc}$ & 44,3 abcdefg \\
\hline REC 2 & $15,3 \mathrm{bc}$ & 32,7 abcdefg \\
\hline Hermes & $17,3 \mathrm{bc}$ & 51,0 abcdefg \\
\hline Baraka & $17,3 \mathrm{bc}$ & $58,0 \mathrm{abcd}$ \\
\hline $\mathrm{CH} 2$ & $18,3 \mathrm{bc}$ & $13,3 \mathrm{~g}$ \\
\hline Santé & $18,7 \mathrm{bc}$ & $14,7 \mathrm{fg}$ \\
\hline BACH 5 & $19,7 \mathrm{abc}$ & 42,0 abcdefg \\
\hline BACH 1 & $20,3 \mathrm{abc}$ & 52,7 abcde \\
\hline Ranger Russet & $20,7 \mathrm{abc}$ & $68,0 \mathrm{a}$ \\
\hline Pira 25 & $20,7 \mathrm{abc}$ & 50,0 abcdefg \\
\hline Daresa & $21,0 \mathrm{abc}$ & $64,7 \mathrm{ab}$ \\
\hline REC 4 & $22,7 \mathrm{abc}$ & 26,3 cdefg \\
\hline MAC 2 & $23,3 \mathrm{abc}$ & 47,0 abcdefg \\
\hline Agria & $26,0 \mathrm{abc}$ & 52,5 abcde \\
\hline REC 3 & $26,3 \mathrm{abc}$ & $62,7 \mathrm{abc}$ \\
\hline HPC 5B & $27,7 \mathrm{abc}$ & 50 abcdefg \\
\hline HPC 13R & $30,3 \mathrm{abc}$ & 29,7 bcdefg \\
\hline BACH 4 & $30,7 \mathrm{abc}$ & 38,3 abcdefg \\
\hline HPC 6R & $31,0 \mathrm{abc}$ & 40,3 abcdefg \\
\hline Omega & $35,0 \mathrm{abc}$ & 47,3 abcdefg \\
\hline Achat & $45,0 \mathrm{ab}$ & 42,3 abcdefg \\
\hline NYL 235-4 & 59,0 a & $64,3 \mathrm{ab}$ \\
\hline $\mathrm{CV}(\%)$ & 21,76 & 27,43 \\
\hline
\end{tabular}

*Médias seguidas pela mesma letra na coluna não diferem significativamente entre si pelo teste de Tukey, $\mathrm{p}<0,05 ;{ }^{1}$ Dados originais; ${ }^{2}$ Dados originais; Para análise, os dados foram transformados em $\log \mathrm{x}$ (*means followed by the same letter in the column do not differ significantly from each other by the Tukey test, $\mathrm{p} \leq 0.05 ;{ }^{1}$ Original data; ${ }^{2}$ Original data; For statistical analysis, data were transformed to $\log \mathrm{x}$ ).

$\mathrm{cm}^{2}$ ), que obteve o maior número de ovos. Estes resultados não confirmam os obtidos no teste com chance de escolha, pois naquele, HPC 6R teve baixo número de ovos, comportando-se como pouco ovipositado. Segundo Lara (1991), uma variedade pode mostrar-se resistente quando comparada com outras em teste com chance de escolha, porém, quando submetida a um teste de obrigatoriedade, ou seja, quando o inseto não tem escolha, a variedade pode acabar revelando-se suscetível. Silva et al. (2008) também relataram que a menor oviposição de $B$. tabaci biótipo $B$ em teste com chance de escolha nos genótipos'Achat', 'Ibituaçu', 'Panda', IAC-1966 e 'Agata' não se manteve em teste sem chance de escolha.

Desenvolvimento ovo-adulto - Não se verificou diferença significativa para a duração do desenvolvimento ovo- -adulto de B. tabaci biótipo B entre os clones de batata (Tabela 3), com médias variando de 34,4 a 36,6 dias, a $19,5^{\circ} \mathrm{C}$. A detecção de diferenças significativas no tempo de desenvolvimento dessa mosca-branca em diferentes genótipos de uma cultura pode ocorrer em genótipos silvestres, como em tomateiro (Baldin et al., 2005) ou mesmo entre germoplasma cultivado, como em aboboreiras (Alves et al., 2005). Todavia, para outras culturas, como melão (Coelho et al., 2009) e batata (Silva et al., 2008), não foram reportadas diferenças no ciclo ovo-adulto desse inseto.

Quanto à emergência de adultos (Tabela 3), BAP 82 destacou-se com o menor índice de adultos emergidos (38\%), contrastando com o Clone APTA 2.135, que teve a maior emergência (80\%). A menor emergência no clone BAP 82 sugere a ocorrência de resistência do tipo antibiose, fato também observado em aboboreira (Alves et al., 2005) e meloeiro (Coelho et al., 2009) em relação a essa mosca-branca. Le Roux et al. (2008) verificaram que a antibiose de genótipos silvestres de batata com origem em S. chomatophilum a Myzus persicae está associada a glicosídeo esteroidal e fenóis. É possível que, no presente estudo, a menor emergência de adultos possa estar associada a essas substâncias químicas, uma vez que ambos os insetos são sugadores, com comportamento alimentar semelhante; contudo, há necessidade de estudos específicos para determinar a causa dessa resistência, analisando-se qualitativa e quantitativamente substâncias desses grupos no clone BAP 82.

Análises de correlação - As análises de correlação simples entre as quatro variáveis estudadas (atratividade, oviposição, tricomas glandulares e tricomas simples) só foi significativa para a interação ovo-adulto $(r=0,67)$, comprovando resultados obtidos com essa mosca-branca em outras culturas em que a maior atratividade corresponde à maior oviposição. Valle \& Lourenção (2002) encontraram correlação significativa positiva para número de ovos e número de adultos de $B$. tabaci biótipo B em soja. Silva et al. (2008) também encontraram correlação significativa positiva para essas duas variáveis em 
Tabela 3. Médias de período de desenvolvimento de ovo a adulto, emergência de adultos e oviposição (ovos $/ 10 \mathrm{~cm}^{2}$ ) de Bemisia tabaci biótipo B, em nove genótipos de batata em casa de vegetação (means of oviposition (eggs/10 cm) and development from egg to adult (days), adult emergence (\%) and ${ }^{2}$ ) of $\mathrm{B}$. tabaci biotype B on nine potato genotypes in no choice test in the greenhouse). Campinas, Instituto Agronômico, 2010.

\begin{tabular}{lccc}
\hline Genótipo & $\begin{array}{c}\text { Número de ovos } \\
\left(\mathbf{n}^{\mathbf{0}} \mathbf{1 0} \mathbf{~ c m}^{\mathbf{2}} \mathbf{( 1 )}^{\mathbf{1})}\right.\end{array}$ & $\begin{array}{c}\text { Desenvolvimento de } \\
\text { ovo a adulto (dias) }\end{array}$ & $\begin{array}{c}\text { Emergência de } \\
\text { adultos } \mathbf{( \% )} \mathbf{~}^{\mathbf{2}}\end{array}$ \\
\hline BAP 82 & $13,5 \mathrm{ab}$ & $36,6 \mathrm{a}$ & $38 \mathrm{~d}$ \\
BACH 4 & $2,5 \mathrm{c}$ & $36,4 \mathrm{a}$ & $47 \mathrm{~cd}$ \\
Baraka & $4,2 \mathrm{bc}$ & $36,4 \mathrm{a}$ & $48 \mathrm{~cd}$ \\
HPC 5B & $6,0 \mathrm{abc}$ & $35,6 \mathrm{a}$ & $58 \mathrm{bc}$ \\
HPC 9B & $3,0 \mathrm{c}$ & $35,6 \mathrm{a}$ & $62 \mathrm{abc}$ \\
HPC 6R & $15,5 \mathrm{a}$ & $35,6 \mathrm{a}$ & $63 \mathrm{abc}$ \\
Achat & $5,3 \mathrm{bc}$ & $35,2 \mathrm{a}$ & $70 \mathrm{ab}$ \\
NYL 235-4 & $7,9 \mathrm{abc}$ & $34,6 \mathrm{a}$ & $73 \mathrm{ab}$ \\
Clone APTA 2135 & $5,6 \mathrm{abc}$ & $34,4 \mathrm{a}$ & $80 \mathrm{a}$ \\
\hline CV (\%) & 31,41 & 3,67 & 15,3 \\
\hline
\end{tabular}

*Médias seguidas pela mesma letra na coluna não diferem significativamente entre si pelo teste de Tukey, $\mathrm{p}<0,05$ (means followed by the same letter in the column do not differ significantly from each other by the Tukey test, $\mathrm{p} \leq 0.05)$; ${ }^{1}$ Dados originais; Para análise, os dados foram transformadas em $\sqrt{\mathrm{x}} ;{ }^{2}$ Dados originais ( ${ }^{1}$ original data; For statistical analysis, data were transformed to $\sqrt{\mathrm{x}} ;{ }^{2}$ original data).

genótipos de batata.

Com relação à densidade dos tricomas glandulares e simples, Silva et al. (2008) verificaram que sua correlação com a atratividade e oviposição de $B$. tabaci biótipo B não é significativa, ou seja, os tricomas não afetam estas variáveis de maneira negativa ou positiva.

Considerando-se os três experimentos, destacam-se como os mais resistentes a $B$. tabaci biótipo $\mathrm{B}$ os clones 'Baraka' e BACH 4, pela baixa atratividade para adultos, menor preferência para oviposição e baixa emergência de adultos. O clone BACH 4 é oriundo de $S$. chacoense, sendo que há casos relatados de resistência de clones de batata oriundos dessa espécie a insetos (Souza et al., 2008); com os resultados do presente trabalho fica comprovada resistência também a $B$. tabaci biótipo B. A utilização desses dois genótipos pode ser indicada para programas de melhoramento genético que visem o desenvolvimento de clones resistentes a $B$. tabaci biótipo B e, no caso de 'Baraka', que é um clone de boas características agronômicas, para experimentação de campo a fim de validar a resistência a essa mosca-branca observada em condições de cultivo protegido.

\section{AGRADECIMENTOS}

À FAPESP, pela concessão de bolsa de mestrado ao primeiro autor, e ao $\mathrm{CNPq}$, pela concessão de bolsa de produtividade em pesquisa ao segundo autor.

\section{REFERÊNCIAS}

ALVES AC; LOURENÇÃO AL; MELO AMT. 2005. Resistência de genótipos de aboboreira a Bemisia tabaci (Genn.) biótipo B (Hemiptera: Aleyrodidade). Neotropical Entomology 34: 973-979.

BALDIN ELL; VENDRAMIM JD; LOURENÇÃO AL. 2005. Resistência de genótipos de tomateiro à mosca-branca Bemisia tabaci (Gennadius) biótipo B (Hemiptera: Aleyrodidae). Neotropical Entomology 34: 435-441.

BALDIN ELL; BENEDUZZI RA; SOUZA DR. 2009. Resistência de genótipos de abobrinha a Bemisia tabaci (Genn.) biótipo B (Hemiptera: Aleyrodidae). Neotropical Entomology 38: 526-530.

BELLOTTI AC; ARIAS B. 2001. Host plant resistance to whiteflies with emphasis on cassava as a case study. Crop Protection 20: 813-823.

BROWN JK. 1994. Current status of Bemisia tabaci as a plant pest and virus vector in agroecosystems worldwide. FAO Plant Protection Bulletin 42: 3-32.

BROWN JK; COATS SA; BEDFORD ID; MARKHAM PG; BIRD J; FROHLICH DR.
1995. Characterization and distribution of esterase electromorphs in the whitefly, Bemisia tabaci (Genn.) (Homoptera: Aleyrodidae). Biochemical Genetics 33: 205-214.

CHU CC; FREEMAN TP; NATWICK ET; BUCKNER JS; NELSON DR; HENNEBERRY TJ. 2000. Bemisia argentifolii adult, nymph and egg densities and egg distribution on selected upland cottons. Journal of Entomology Science 35: 39-47.

COELHO SAMP; LOURENCÃO AL; MELO AMT; SCHAMMASS EA. 2009. Resistência de meloeiro a Bemisia tabaci biótipo B. Bragantia 68: 1025-1035.

COOPER SG; DOUCHES DS; GRAFIUS EJ. 2009. Combining engineered resistance, avidin and natural resistance derived from Solanum chacoense Bitter to control Colorado potato beetle (Coleoptera: Chrysomelidae). Plant Resistance 102: 1270-1280.

FERNANDES MES; SILVA DJH; FERNANDES FL; PICANÇO MC; GONTIJO PC; GALDINO TVS. 2009. Novos acessos de tomateiro resistentes à mosca-branca biótipo B. Pesquisa Agropecuária Brasileira 44: 1545-1548.

FONTES FVHM; COLOMBO CA; LOURENÇÃO AL. 2010. Caracterização molecular e divergência genética de Bemisia tabaci (Genn.) (Hemiptera: Aleyrodidae) em diferentes culturas e locais de cultivo. Neotropical Entomology 39: 221-226.

FRANÇA F, VILLAS BÔAS GL, CASTELO BRANCO M. 1996. Ocorrência de Bemisia argentifolii Bellows \& Perring (Homoptera: Aleyrodidae) no Distrito Federal. Anais da Sociedade Entomológica do Brasil 25: 369-372

JIANG YX, NOMBELA G, MUÑIZ M. 2001. Probing and feeding behavior of two distinct biotypes of Bemisia tabaci (Homoptera: Aleyrodidae) on tomato plants. Entomologia Experimentalis et Applicata. 92: 357-366.

KNOW M; HYEON-MOOK C; YOUNG-JOON A. 2006. Relationship between feeding damage beet armyworm Spodoptera exigua (Lepidoptera: Noctuidae) and leaf trichome density of potato. Korean Journal of Applied Entomology 9: 361-367.

LARA FM. 1991. Princípios de resistência de plantas a insetos. 2 ed. São Paulo: ED. Ícone. $336 \mathrm{p}$.

LARA FM; CORBO A; FILGUEIRA LK; STEIN CP. 2004a. Resistência de genótipos de batata ao pulgão. Horticultura Brasileira. 22: 775-779.

LARA FM.; SCARANELLO AL.; BALDIN ELL.; BOIÇA JUNIOR AL; LOURENÇÃO AL. 2004b. Resistência de genótipos de batata a larvas e adultos de Diabrotica speciosa. Horticultura Brasileira 22: 761-765.

LE ROUX V; DUGRAVOT S; CAMPAN E; FRANÇOISE D; VICENTE C; GIORDANENGO P. Type of aphid resistance in wild Solanum. 2008. Journal of Economic Entomology 101: 584-591.

LIMA ACS; LARA FM. 2001. Mosca-branca (Bemisia tabaci): morfologia, bioecologia e controle. Jaboticabal. p. 1-76.

LOURENÇÃO AL. 1980. Bemisia tabaci 
(Gennadius, 1889) (Homoptera: Aleyrodidae) em soja (Glycine max (L.) Merrill): influência da variedade, da idade da planta e de cruzamentos intervarietais sobre a oviposição e desenvolvimento do inseto. Piracicaba, SP: ESALQ/USP. 58 p. (Dissertação mestrado).

LOURENÇÃO AL; NAGAI H. 1994. Surtos populacionais de Bemisia tabaci no estado de São Paulo. Bragantia 53: 53-59.

LOURENÇÃO AL; SOUZA-DIAS JAC; FUGI CGQ. 2003. Mosca branca: superpraga da agricultura mundial ameaça a bataticultura brasileira. Batata Show 6: 7-8.

MANSARAY A; SUNDUFU AJ. 2009. Oviposition, development and survivorship of the sweetpotato whitefly Bemisia tabaci on soybean, Glycine max, and the garden bean, Phaseolus vulgaris. Journal of Insect Science 9: 1-6.

MEDEIROS AH; TINGEY WM. 2006. Glandular trichomes of Solanum berthaultii and its hybrids with Solanum tuberosum affect nymphal emergence, development, and survival of Empoasca fabae (Homoptera: Cicadellidade). Journal of Economic Entomology 99: 1483-1489.

PAINTER RH. 1968. Insect resistance in crop plants. New York: Mac Millan. 520p.

RAIJ B; CANTARELLA H; QUAGGIO JA; FURLANI AMC. 1997. Recomendações de adubação e calagem para o estado de São Paulo. Campinas: Instituto Agronômico. Boletim Técnico 100, 285 p.

SILVA MS; LOURENÇÃO AL; SOUZA-DIAS JAC; MIRANDA FILHO HS; RAMOS VJ; SCHAMMASS EA. 2008. Resistance of potato genotypes (Solanum spp.) to Bemisia tabaci biotype B. Horticultura Brasileira 26: 221-226.

SOUZA-DIAS JAC; SAWASAKI HE; SILVA MS; GIUSTO AB. 2005. Mosca-branca (Bemisia tabaci) $\mathrm{x}$ viroses na bataticultura: não bastava o mosaico amarelo deformante (geminivírus - TYVSV) e agora também o enrolamento da folha (luteovírus - PLRV)? Batata Show 12: 13.

SOUZA VQ; PEREIRAAS; FRITCHE NETO R; SILVA GO; OLIVEIRA AC. 2005. Potential of selection among and within potato clonal families. Crop Breeding and Applied Biotechnology 5: 199-206.

SOUZA VQ, PEREIRA AS, SILVA GO; FRITCHE NETO R; CASTRO CM. 2008. Avaliação de genótipos de batata selecionados para resistência a insetos-praga. Boletim de pesquisa e desenvolvimento 65. Pelotas: EMBRAPA Clima Temperado, 29p.

VAN LENTEREN JC; NOLDUS LPJJ. 1990. Whitefly-plant relationships: behavioral and ecological aspects. In: GERLING D (ed). Whiteflies: their bionomics, pest status and management. Andover: Intercept. p.47-89.

VALLE GE; LOURENÇÃOAL. 2002. Resistência de genótipos de soja a Bemisia tabaci (Genn.) biótipo B (Hemiptera: Aleyrodidade). Neotropical Entomology 3: 285-295. 